Commun. math. Phys. 37, 221-256 (1974)

(C) by Springer-Verlag 1974

\title{
Generalized Quantum Mechanics
}

\author{
Bogdan Mielnik \\ Institute of Theoretical Physics, Division of Mathematical Physics, Warszawa, Poland
}

Received June 15, 1973

\begin{abstract}
A convex scheme of quantum theory is outlined where the states are not necessarily the density matrices in a Hilbert space. The physical interpretation of the scheme is given in terms of generalized "impossibility principles". The geometry of the convex set of all pure and mixed states (called a statistical figure) is conditioned by the dynamics of the system. This provides a method of constructing the statistical figures for non-linear variants of quantum mechanics where the superposition principle is no longer valid. Examples of that construction are given and its possible significance for the interrelation between quantum theory and general relativity is discussed.
\end{abstract}

\section{Introduction}

In turn of development of quantum theory efforts were made to present a geometric description of quantum mechanics independent of "wave functions" and "complex amplitudes". The best known such description was originated by Birkhoff and von Neumann [2] and completed by Piron [18]. It explores a partial order relation in a idealized set of "yes-no measurements" called a "quantum logic". The resulting approach though mathematically profound is not physically complete. In the last ten years two other approaches have been developed. One is the algebraic approach reflecting the physics of operations which can be performed on statistical ensembles. This aspect has been introduced to axiomatic quantum field theory by Haag and Kastler [10] and it reappears as the main motif in the present day quantum statistics. The other approach, originated already in the fourties (Segal [1]) might be called "convex". It explores the convex structures of quantum mechanics with a special attention concentrated on the convex set of all states (pure and mixed) of a quantum system. The description of quantum mechanics from that point of view was most systematically explored by Ludwig [14] and further developed in $[3-6,11,15-17,19,21,22]$; it now becomes one of main currents in the foundation of quantum theory. The synthesis of the convex and the algebraic approaches has been gradually achieved $[3,5,6,9-11,16,19]$. It brought the complete geometrization of quantum mechanics including the description of the present day formalism of 
Hilbert spaces in terms of physically meaningful axioms $[9,14,15,19,21]$, the general classification of the operation $[6,11]$, the definition of filters as endomorphism of a convex cone of beams $[3,16]$ and finally, the construction of the transition probabilities as affine geometric invariants of a convex set [16]. As a result of that development a generalized convex scheme of quantum mechanics has emerged from the point of view of which the scheme of the present day theory is not unique but is a particular member of a vast family of "quantum worlds" mathematically admissible. The conjecture was also rised that the convex set theory might play a similar role in quantum physics as the Riemannian geometry in general relativity [16]. The aim or the present paper is to take the next step by showing that the "convex scheme" is flexible enough to comprise nonlinear versions of quantum mechanics in which a non-linear wave equation would play the role of the Schrödinger equation. With this aim the geometric description of quantum mechanics based on the convex set theory is outlined in $\S 2$. In $\S 3$ and $\S 4$ the geometry of a system is related with the dynamics which allows the construction of the convex manifolds of quantum states for systems obeying a generalized wave mechanics. Some applications of the resulting scheme are indicated in $\S 4$ and its relation to other physical theories is discussed in $\S 5$.

\section{Convex Scheme (Outline) ${ }^{1}$}

The elements of convex set theory are rooted in primitive concepts of quantum mechanics. The most fundamental such concept is that of a quantum state. Given a statistical ensemble of objects of any nature, the state is the collection of the physical properties of an average ensemble individual. For the above notion of a state the following concept of a mixture becomes natural. Given certain ensembles $\mathscr{E}_{1}, \ldots, \mathscr{E}_{n}$ corresponding to states $x_{1}, \ldots, x_{n}$ and given numbers $p_{1}, \ldots, p_{n} \geqq 0, p_{1}+\cdots$ $\cdots+p_{n}=1$, one can form a new ensemble $\mathscr{E}$ of which a fraction $p_{j}$ comprises randomly chosen objects of $\mathscr{E}_{j}(j=1, \ldots, n)$ : the ensemble $\mathscr{E}$ defines a new state $x$ which will be denoted $x=p_{1} x_{1}+\cdots+p_{n} x_{n}$ and called a mixture of $x_{1}, \ldots, x_{n}$. The concept of a mixture induces that of a pure state: a state is called pure if it cannot be represented as a mixture (with all coefficients non-vanishing) of any physically distinct states. These definitions suggest that the set of all states of a physical object should possess the structure of a convex set. Some definitions concerning that structure are given below.

Definitions. A convex set is a subset of an affine space containing together with any two points the interval joining them. Here, an affine

\footnotetext{
1 This section is a review of essentially known material.
} 
space is any set $E$ of elements called points with a linear combination operation assining to each finite system of points $x_{1}, \ldots, x_{n} \in E(n=1,2, \ldots)$ and any system of numbers $\lambda_{1}, \ldots, \lambda_{n} \in \mathbb{R}, \lambda_{1}+\cdots+\lambda_{n}=1$ a new point $\lambda_{1} x_{1}+\cdots+\lambda_{n} x_{n}$; the linear operation has properties which allow one to represent $E$ as a plane in a real linear space. An affine topological space is an affine space $E$ with a topology in which the linear operation is continuous. Given an affine space $E$ and a system of points $x_{1}, \ldots, x_{n} \in E$, any linear combination $p_{1} x_{1}+\cdots+p_{n} x_{n}$ with $p_{1}, \ldots, p_{n} \geqq 0, p_{1},+\cdots$ $\cdots+p_{n}=1$ is called a convex combination of $x_{1}, \ldots, x_{n}$. For $E$ an affine topological space, a continuous analogue of that operation can be introduced. Given a subset $X \subset E$ with the topology induced by that of $E$ and given a positive measure $\mu$ defined on Borel subsets of $X$ such that $\mu(X)=1$ (a probability measure on $X$ ) the integral $\int_{X} x d \mu(x)$, if it exists, is called a convex integral of points $x \in X$ over the measure $\mu$. The convex combination is a special case of a convex integral obtained by taking the measure $\mu$ to vanish outside of a finite set of points. Given an affine space $E$ and two points $x_{1}, x_{2} \in E, x_{1} \neq x_{2}$, the set of all linear combinations $E\left(x_{1}, x_{2}\right)=\left\{\lambda_{1} x_{1}+\lambda_{2} x_{2}: \lambda_{1}, \lambda_{2} \in \mathbb{R}, \lambda_{1}+\lambda_{2}=1\right\}$ is called the straight ine determined by $x_{1}$ and $x_{2}$ while the set of all convex combinations $I\left(x_{1}, x_{2}\right)=\left\{p_{1} x_{1}+p_{2} x_{2}: p_{1}, p_{2} \geqq 0, p_{1}+p_{2}=1\right\}$ is called the straight line interval joining $x_{1}$ and $x_{2}$. Any point of $I\left(x_{1}, x_{2}\right)$ different from the end points $x_{1}$ and $x_{2}$ is called an internal point of $I\left(x_{1}, x_{2}\right)$. Given a convex set $S \subset E$ an element $x \in S$ is called an extremal point of $S$ if it cannot be represented as a convex combination with both coefficients positive of any two distinct points of $S$. Thus, $x$ is extremal if it is not an internal point of any interval $I C S$.

The most general axiom reflecting the phenomenology of mixtures in quantum mechanics can be now formulated as follows. For any quantum system the set of all states can be represented as a closed convex set $S$ in a certain affine topological space $E$. The convex combinations in $S$ correspond to the state mixtures while the extremal points of $S$ represent the pure states of the system. The topology on $S$ reflects the observable properties of quantum states.

Here, physical significance may be attributed to the set $S$ alone: the surroundings affine space $E$ is introduced only as an auxiliary construct ${ }^{2}$. For reasons of economy it will be assumed that $S$ spanns $E$. For quantum mechanical systems which are the objective of this paper it will be assumed in addition that $S$ contains a set of extremal points rich enough to represent any point of $S$ as a convex integral of extremal points. The

${ }^{2}$ One could think about an axiomatic approach where the convex set $S$ would be defined without involving the surrounding space. A step in that direction was recently taken by Gudder [22]. 


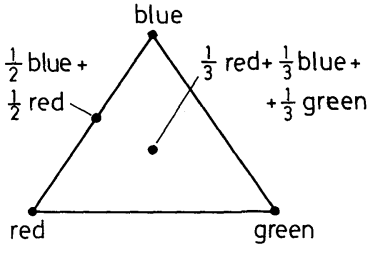

Fig. 1

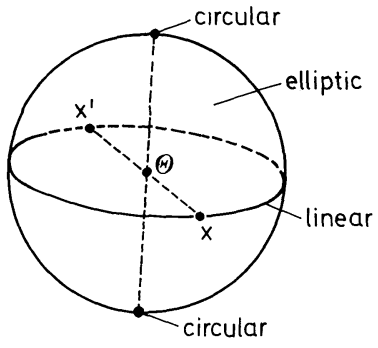

Fig. 2

convex set $S$ plays a fundamental role in quantum statistics; it will be further called a statistical figure (see also [16]). Two simple examples of that structure are given below.

Figure 1 represents the mixtures which can be formed of classical objects of three types (for example, red, green and blue balls). The statistical figure here is a triangle in a 2-dimensional affine space: the vertices are pure states corresponding to one-colour ensembles, while the remaining points represent the mixed states with one mixed state (the center) distinguished (completely random mixture). A similar structure can be obtained by considering mixtures of classical objects of $n$ possibles types: in that case the statistical figure is a simplex with $n$ vertices in an $(n-1)$-dimensional affine space. The case of $n=\infty$ is essential for realistic models of classical mechanics. Here, the pure states correspond to points of a classical phase space $P$ (endowed with a certain natural topology) and the statistical figure $S$ is the convex set of all probability measures on $P$ with the topology induced by that of $P$. The convex set of all probability measures on a certain topological space is a generalized simplex whose vertices are all point-concentrated measures. It is an important property of the simplexes that each point of a simplex can be uniquely represented as a convex integral of extremal points. This fact reflects the classical nature of the corresponding objects: it is a crucial feature of classical objects that their statistical ensembles can be uniquely decomposed into the pure components. Thus, the simplexes have to be considered the statistical figures of classical theories.

A different example is shown in Fig. 2, which represents the polarization states of a photon. Here, the statistical figure is an ellipsoid in a 3-dimensional affine space. The surface of the ellipsoid represents the pure polarization states: the equator comprises the linear polarizations, the poles are the circular polarization states and the remaining points of the surface correspond to the elliptic polarizations. Each two antipodes 
of the ellipsoid correspond to the "opposite" polarizations (for instance, each two antipodes on the equator represent two mutually orthogonal linear polarizations). The internal points of the ellipsoid stand for the mixed states with one mixed state $\theta$ (the centre) distinguished (polarization chaos). For the above statistical figure the decomposition of mixtures into their pure components is no longer unique: each mixed state can be represented in many ways as a combination of pure states. Thus, the chaos state can be represented as $\theta=\frac{1}{2} x+\frac{1}{2} x^{\prime}$ where $x$ and $x^{\prime}$ are any two antipodes of the ellipsoid. Physically, this means that having a light beam in the polarization state $\theta$ one cannot say whether the beam has been prepared by mixing two linearly polarized beams or by mixing two circularly polarized beams or in any other way. This fact illustrates a certain general "impotence law" coded in the geometry of $S$.

\section{Principle of Impossibility}

The law which emerges from the example in Fig. 2 might be given the following form. Having a mixed statistical ensemble of non-classical objects one cannot determine uniquely its pure components and find out how the mixture has been prepared. Two mixtures created in two distinct ways by taking different collections of pure states may be physically indistinguishable. This statement is one of the most general negative laws limiting the perception of quantum ensembles: it might be called the first principle of impossibility of quantum theory and considered the main manifestation of the non-classical nature of microobjects. The above law is not exclusive for the orthodox theory but it can be read from the geometry of any statistical figure $S$ which is not a simplex (see Fig. 3). A non-simplicial shape of $S$ is a geometric expression of nonclassical character of the corresponding objects.

"First impossibility":

$$
x=p_{1} x_{1}+p_{2} x_{2}
$$$$
x=q_{1} y_{1}+q_{2} y_{2} \text {. }
$$

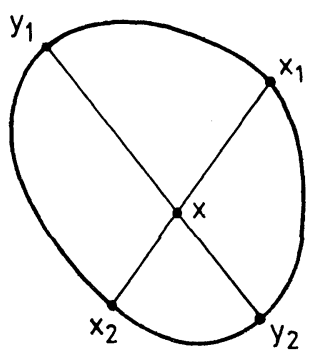




\section{Counters}

Though it was known for a long time that the shape of $S$ reflects the physics of the corresponding quanta, it was only recently discovered that this shape contains the complete information concerning the properties of quantum states. In reading that information the following concept of a normal functional is essential.

Definition. Given an affine space $E$, a functional $\phi: E \rightarrow \mathbb{R}$ is called linear if $\phi\left(\lambda_{1} x_{1}+\cdots+\lambda_{n} x_{n}\right)=\lambda_{1} \phi x_{1}+\cdots+\lambda_{n} \phi x_{n}$ for any $x_{1}, \ldots, x_{n} \in E$, $\lambda_{1}, \ldots, \lambda_{n} \in \mathbb{R}, \lambda_{1}+\cdots+\lambda_{n}=1$. Given a convex set $S$ in an affine topological space $E$, a linear continuous functional $\phi: E \rightarrow \mathbb{R}$ is called normal on $S$ if $0 \leqq \phi x \leqq 1$ for every $x \in S$.

The normal functionals admit a simple geometric representation. Any non-constant linear continuous functional $\phi$ in an affine topological space is completely determined by a pair of closed parallel hyperplanes on which it takes the value 0 and 1. Now, $\phi$ is normal on $S$ if the set $S$ lies in between the hyperplanes $\phi=0$ and $\phi=1$ (see Fig. 4).

If the convex set $S$ is a statistical figure for a certain physical system, the normal functionals possess a natural physical interpretation. A. meaningful theory besides physical objects describes also measuring devices. The typical measuring device of quantum theory is a particle counter. Given a counter $\phi$ and a state $x \in S$ the number $\phi x$ will mean the average fraction of systems in $x$-state detected by the counter $\phi(0 \leqq \phi x \leqq 1)$. Since counters considered here react only to individual systems of the ensemble (without taking into account the interrelations between them), it follows that for any mixed ensemble the total number of particles (systems) detected is the sum of the corresponding numbers for all the components of the mixture. This leads to: $\phi\left(p_{1} x_{1}+\cdots+p_{n} x_{n}\right)$

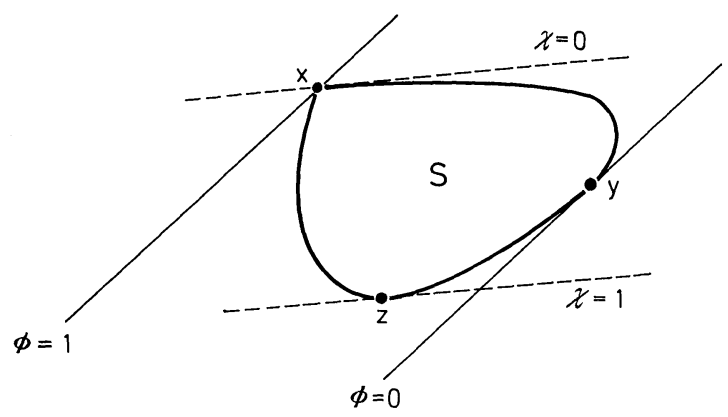

Fig. 4 
$=p_{1} \phi x_{1}+\cdots+p_{n} \phi x_{n}$ and so, each counter defines a certain normal functional on the statistical figure $S$ (and thus, on the whole of $E$ ). The question now arises: how rich is the set of all normal functionals which correspond to certain physical counters? As no counter-example is known, it will be assumed that each normal functional represents a way of detecting a property of the system which, at least in principle, could be realized by constructing an adequate counter. For example, if the convex set in Fig. 4 were a statistical figure for certain physical particles, the pair of planes $\phi=0$ and $\phi=1$ would represent a counter registering unmistakenly all particles in state $x$ (" $x$-particles") and blind to all $y$-particles, whereas the planes $\chi=0$ and $\chi=1$ would represent a counter registering all $z$-particles, $1 / 2$ of the $y$-particles and blind to $x$-particles. Since for the convex set of quite arbitrary shape the non-trivial normal functionals might not exist, some general assumptions as to the structure of $S$ are still necessary. What will be assumed below is that the shape of $S$ allows the existence of a class of normal functionals rich enough to distinguish the points of $S$ : the convex set with that property is called bounded.

The assumptions up to now can be summarized as follows. For any quantum system the set of all states is a closed and bounded convex set $S$ (called a statistical figure) in an affine topological space E. (The physically essential structure here is $S$ while the surrounding affine space $E$ is spanned by $S$ as an auxiliary construct.)

The statistical figure $S$ uniquely determines the class of normal functionals represented by all pairs of parallel hyperplanes enclosing $S$ : every normal functional corresponds to a quantum mechanical counter which might be used to test the system properties.

On these assumptions, all the physical information contained in the geometry of $S$ may be now decoded. One of the most familiar such information is the "quantum logic" of Birkhoff, von Neumann, and Piron $[2,18,12]$.

\section{Quantum Logic}

In the geometry of convex sets the following concept of a wall is of importance.

Definition. Given a convex set $S$, a wall of $S$ is any convex subset $S^{\prime} \subset S$ such that, whenever $S^{\prime}$ contains an internal point of any straight line interval $I \subset S$, it must also contain the total interval $I$. Thus, $S^{\prime}$ is a wall if the following implications hold: 1) $x_{1}, x_{2} \in S^{\prime}, p_{1}, p_{2} \geqq 0, p_{1}+p_{2}$ 
$=1 \Rightarrow p_{1} x_{1}+p_{2} x_{2} \in S^{\prime}$; 2) $x_{1}, x_{2} \in S, p_{1} x_{1}+p_{2} x_{2} \in S^{\prime}$ with $p_{1}, p_{2}>0$, $p_{1}+p_{2}=1 \Rightarrow x_{1}, x_{2} \in S^{\prime}$.

The above concept of a wall generalizes that of an extremal point: the extremal points are simply one-point walls of $S$. Geometrically, a wall can be associated with an intuitive idea of a "maximal plane fragment" (of any dimensionality) of the boundary of $S$. Each convex set has at least two inproper walls: the empty set $\varnothing$ and whole of $S$. For any convex set $S$ the walls form a partially ordered set with the ordering relation $\leqq$ meaning set theoretical inclusion. As is immediately seen, the common part of any family of walls is again a wall: hence, the walls form also a lattice (where for any family of walls the intersection of all upper bounds defines the lowest upper bound). If $S$ is a statistical figure this lattice admits a natural physical interpretation. It is an old question whether the formalism of quantum theory is adequate to describe the properties of single systems. What is verified directly in the most general quantum experiment are rather the properties of statistical ensembles. However, the properties of single systems can be introduced by an abstraction process $[12,16]$. One can agree, that a property $P$ of quantum ensembles defines a property of single systems provided that it is "additive" and "hereditary" 3 : if any two ensembles have the property $P$ their mixtures must have it too. Conversely, if any mixed ensemble has the property $P$, so must have each of the mixture components. These conditions mean that the subset of all states with the property $P$ should be a wall of $S$. One thus guesses that the lattice of walls of $S$ represents the set of all possible physical properties of a single system ordered according to their generality. In axiomatic approaches to quantum mechanics an important role is attributed to the notion of completely excluding properties. This notion finds a simple geometric description too. Given two walls (properties) $S_{1}, S_{2} \subset S$ and a normal functional $\phi$, it will be said that $\phi$ completely separates $S_{1}$ and $S_{2}$ if either $\phi x_{1}=0$, and $\phi x_{2}=1$ or $\phi x_{1}=1$ and $\phi x_{2}=0$ for every $x_{1} \in S_{1}$ and $x_{2} \in S_{2}$ (i.e. if the corresponding counter is completely blind to the particles with one of these properties and detects all the particles with the other). Now, two properties (walls) are called excluding or orthogonal $\left(S_{1} \perp S_{2}\right)$ if there exists at least one counter completely separating them. The set of all physical properties of a microparticle with the relations of inclusion $\leqq$

3 Not every property of an ensemble is of such a nature that it can be attributed to each single ensemble individual. An example can be obtained by considering a particle beam and a semi-transparent window: the fact that the average beam particle penetrates the window with probability $1 / 2$ reflects a certain property of the beam as a whole. This property does not necessarily concern each single beam particle: for it may happen that the beam is a mixture of two distinct types of particles one of which is completely transmitted and the other completely absorbed by the window. 
and exclusion $\perp$ is what one traditionally calles a logic of the particle or quantum logic 4 .

Hence, in the present approach the quantum logic is no longer a fundamental structure but one of the particular aspects of the geometry of $S$.

\section{Second Principle of Impossibility}

Besides the structure of the "logic" the geometry of $S$ allows one to read a numerical relation between pure states generalizing the basic invariant $|(\psi, \varphi)|^{2}$ of the orthodox theory. Let $x$ and $y$ be two extremal points of a statistical figure $S$ and let $Q(y)$ denote the set of all normal functionals taking the value 1 at $y$. If $x \perp y$, there exists in $Q(y)$ at least one functional vanishing at $x$. In general, however, such a functional in $Q(y)$ may not exist because of the geometry of $S$. An example of this situation is shown on Fig. 5 .

For the convex set here each point of the $\widehat{\operatorname{arc}} \widetilde{z_{1} z_{2}}$ is orthogonal to the extremal point $y$ : suitable separating functionals are determined by all possible pairs of parallel support lines $P_{j}, P_{j}^{\prime}$ one of which supports $S$ at $y$ and the other at an arbitrary point of $\widetilde{z_{1}} z_{2}$. A similar separating functional, however, does not exist for the pair of points $x$ and $y$ as there is no parallel pair of straight lines supporting $S$ at $x$ and $y$. As seen on Fig. 5 the smallest value possible at $x$ for the functionals of the family $Q(y)$ is $1 / 2$ and is accepted by the functional $\phi$ represented by the pair of lines $P_{1}, P_{1}^{\prime}$. Thus, if the convex set in Fig. 5 were a statistical figure for certain physical quanta, it could be infered that no counter can be constructed registering all $y$-quanta and less than the average fraction $1 / 2$ of $x$-quanta. This illustrates a certain general impossibility law coded in the geometry of the statistical figure. In order to formulate it more precisely the following geometric quantity is needed.

Definition. Given a closed convex set $S$ and a pair of extremal points $x, y \in S$ the ratio $x: y$ is the lower limit at $x$ of all normal functionals

${ }^{4}$ The so introduced concept of a logic is wider than in the majority od axiomatic approaches. The notion of orthogonality employed here is more primitive than the usually introduced concept of negation. Given the lattice $W$ of walls with the orthogonality $\perp$, the physically interpretable negation can or cannot be introduced dependingly on the structure of the relations $\perp$ and $\leqq$ on $W$. If for an $a \in W$ the family $a^{\perp}=\{x \in W: x \perp a\}$ contains its lowest upper bound, this element can be denoted $a^{\prime}$ and called the "orthogonal complement" (negative) of $a$. If not, then no unique negative can be assigned to $a$. For an arbitrary convex set the resulting logic $W(\leqq, \perp)$, in general, does not admitt the construction of the orthocomplementation $a \rightarrow a^{\prime}$.

It is, an open question whether all the walls of $S$ should be considered the physically essential elements of the "logic" or some regularity requirements should be suplemented (stating, for instance, that only the closed walls of $S$ correspond to "physically verifiable properties"). An extensive discussion of an equivalent problem is due to Giles [8]. 


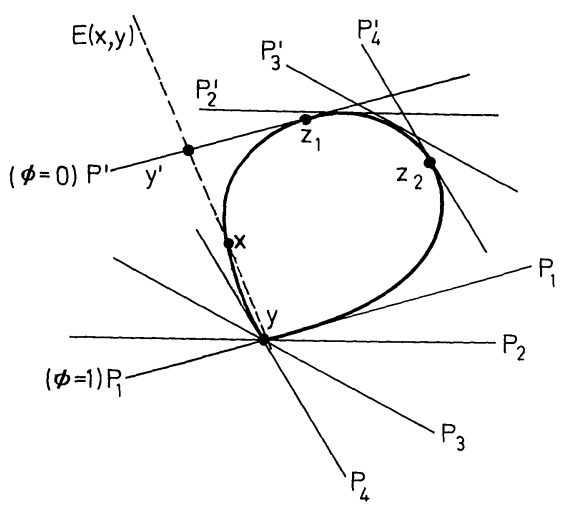

Fig. 5

taking the value 1 at $y$ :

$$
x: y=\inf _{\phi \in Q(y)} \phi x
$$

For any convex set $S$ the ratios of the extremal points are uniquely defined by the shape of $S$ and can be determined by an abstract construction employing support planes [16].

If $S$ is a statistical figure of certain physical systems, the non-vanishing of the quantity (2.1) reflects an inavoidable lack of selectivity of quantum mechanical measurements. Given two pure states $x$ and $y$ no counter can be constructed which detects all $y$-systems and less than a fraction $x: y$ of $x$-systems. More generally, every physical process leading to a certain macroscopic effect for all $y$-systems must inavoidably lead to the same effect for at least $x: y$ of $x$-systems. The above statements form one of essential quantum laws which will be further called the second principle of impossibility. Because of their role in that principle, the ratios of the extremal points of any statistical figure will be called the detection ratios (see also [16]).

A particular form of the "second impossibility" is observed in orthodox quantum mechanics. Here, the statistical figure is the set of all density operators in a certain Hilbert space $\mathscr{H}: S_{\mathscr{H}}=\left\{x \in \mathscr{L}(\mathscr{H}): x^{+}\right.$ $=x \geqq 0, \operatorname{Tr} x=1\}$. The extremal points of $S_{\mathscr{H}}$ are the simple projection operators of the form $|\psi \times \psi|$ where $\psi$ are the unit vectors in $\mathscr{H}$. For an arbitrary pair of extremal points $|\psi \times \psi|$ and $|\varphi \times \varphi|$ the detection ratio has been found in [16]:

$$
|\psi \times \psi|:|\varphi \times \varphi|=|(\psi, \varphi)|^{2}
$$


This fact allows one to deepen the statistical interpretation of the quantities $|(\psi, \varphi)|^{2}$ in orthodox theory. According to the traditional interpretation these quantities possess the following meaning of the transition probabilities. Given any pure state $|\varphi \times \varphi|$ the theory assumes the existence of a measuring device testing for miscrosystems in that state. When this device is applied to an ensemble systems in another state $|\psi \times \psi|$ a fraction $|(\psi, \varphi)|^{2}$ of them, on average, passes the test with the result positive. What may be infered now however, is not merely the existence of this sort of verifying device, but also the basic impossibility of any more selective apparatus. Given two pure states corresponding to unit vectors $\psi, \varphi \in \mathscr{H}$, no particle absorber is possible transparent to all $\varphi$-systems and transmitting less than a fraction $|(\psi, \varphi)|^{2}$ of $\psi$-systems. Generally, no physical process is possible which tolerates all $\varphi$-systems and less than a fraction $|(\psi, \varphi)|^{2}$ of $\psi$-systems. The quantities $|(\psi, \varphi)|^{2}$ establish an absolute selectivity limit for quantum experiments. The above facts, though proved analytically [16] possess a simple geometric meaning. As an illustration consider polarized light beams. If $x$ and $y$ are the states of the circular and linear polarizations of a photon respectively, there exist a device transparent to linear $y$-photons and transmitting ony half of the circular $x$-photons (the Nicol prism with its polarization plane coinciding with that of the $y$-photons). However, no window can exist more selective than the Nicol prism, that is, transparent to linearly polarized photons but absotbing more than a fraction $1 / 2$ of circularly polarized photons. The impossibility of such a device follows immediately from the geometry of the ellipsoid in Fig. 2 (construction with the support planes), whereas the analytical representation of the points of that ellipsoid by density operators in a Hilbert space is much less obvious.

It is worth while to notice that the "second impossibility" exhibits an implicit dynamical contents of the statistical figure. In fact, the assertion about the impossibility of certain physical processes is a genuine dynamical statement limiting a priori the class of evolution processes admissible. This limitation is present on the apparently pre-dynamical level of the theory, as a necessary condition for the applicability of its mathematical language. Thus, in supplementing the structure of quantum states by dynamical equations the precaution must be taken to respect an "innate" dynamical information contained in the geometry of $S$. This seems to be a special case of Haag's general idea about the necessary consistency between the primitive measurement axioms of quantum theory and its mature form of a dynamical theory ${ }^{5}$.

5 The importance of this type of consistency for quantum theory was pointed out to the author by Professor R. Haag during the winter school in Karpacz, February 1968. 


\section{Problem of Generality}

As becomes clear, the convex set theory introduces into quantum mechanics a flexibility similar to that which the Riemannian geometry achieves in the space-time physics. Indeed, it is significant that one can describe the structure of quite an arbitrary convex set in terms of typically quantum mechanical concepts such as the "detection ratios" and the "quantum logic". When the set $S$ deviates from the traditional manifold of "density matrices", those concepts do not loose their sense: they only change the geometry. This allows a consistent description of generalized systems where the geometry of the "transition probabilities" and the structure of "logic" would not be originated by the Hilbert space theory. Thus, one might think about a possibility of non-orthodox "quantum worlds" where the "logical" axioms of Birkhoff, von Neumann and Piron $[2,18]$ would be relaxed and the "operational" axioms of Pool [19] and Gunson [9] would not hold. One might also construct hypothetical systems with the lattice of "properties" being not orthocomplemented and the axiom of Ludwig [14] about the "sensitivity increase of effects" broken. The simplest example embodying all those non-orthodox features is represented by a hypothetical statistical figure of the form of a square in a 2-dimensional affine plane:

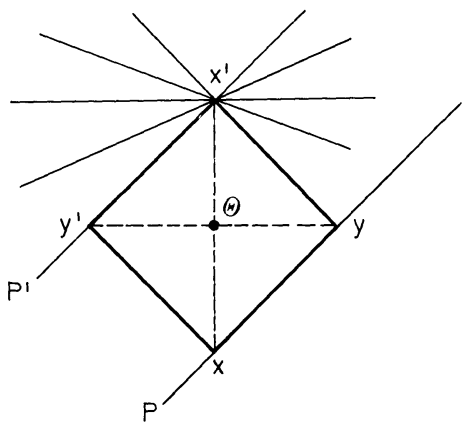

Fig. 6

Here, the pure states are the vertices $x, x^{\prime}, y, y^{\prime}$ and the remaining points of the square are mixed states with one mixed state (the centre) distinguished as the "total chaos". For any pair of pure states there exists a separating normal functional (thus, e.g., the pair of support lines $P, P^{\prime}$ in Fig. 5, defines a normal functional separating $x$ and $y$ from both $x^{\prime}$ and $y^{\prime}$ ). Hence, the detection ratios vanish and there is no second impossibility. Still, the first impossibility is present: the mixtures $\frac{1}{2} x$ $+\frac{1}{2} x^{\prime}$ and $\frac{1}{2} y+\frac{1}{2} y^{\prime}$ are indistinguishable as they both define the "total chaos". The logic of properties forms a Dedekind's lattice (there are 
4 types of walls: the empty set $\varnothing$, the vertices, the sides, and the whole square). However, it is not orthocomplemented: for any pure state $x$, among the walls orthogonal to $x$ no greatest one exists, and so, no unique negation in "quantum logic" can be defined.

A question now arises: is it so, that a statistical figure like that on Fig. 6 could indeed reflect the statistics of some real objects, or it is bound to represent a kind of science fiction structure in the framework of axiomatic quantum mechanics? In spite of known arguments $[9,18,19]$ the answer to that question would be still premature. Instead of looking for plausible axioms limiting the structure of $S$ to that of the orthodox theory it is better to analyse first what what the other structures might bring. A programme for such an investigation has been raised in [16]. In the present paper the "convex scheme" is used in whole generality: it will be shown that only this unrestricted scheme is wide enough to describe the possible non-linearities in quantum mechanics.

\section{Relation to Non-Linearity}

The non-linearity may affect various levels of quantum theory. One can deal with certain physical quanta which, when propagate in vacuum, are well described by linear wave equations: nevertheless their dense clouds (approximately described by $c$-number fields) obey non-linear propagation laws ${ }^{6}$. This kind of non-linearity will be called secondary. One can also think about more basic type of non-linearity affecting not only the macroscopic fields but also the isolated quanta: so, that even a free particle in vacuum would not propagate according to a linear equation. The non-linearity of that kind might be called primary. A hypothetical experiment in which such a phenomenon could be detected can be imagined as follows. Suppose, one has a quantum beam and a pair of slids like those in Young experiment. The beam intensity is low and an analogue of Fabricant experiment is performed: the quanta pass the slids and drop separately onto a screen behind. Suppose now, that the statistics of quantum hits on the screen reveals the interference fringes. However, unlikely to the traditional quantum mechanical experiments, the intensity of the fringes is not a sinusiodal function of the screen cartesian coordinate: the phenomenon, though typically quantum in spirit, fails to fit the scheme of the orthodox quantum mechanics. The question then arises: what sort of quantum mechanics could be constructed on the basis of such a hypothetical experiment? An interesting variant of this question is obtained by assuming that the shape of the interference fringes can be associated with a certain non-linear wave

6 This happens in quantum electrodynamics where each single photon in vacuum, although "dressed", propagates according to Maxwell equations, whereas the intense photon beams exhibit non-linearities because of photon-photon interactions. 
equation. The question then becomes: how can one construct a non-linear analogue of quantum mechanics with a non-linear wave equation playing the role of the Schrödinger equation?

To give an answer, one is led back to the origin of quantum mechanics. The development of that theory involved two basic heuristic steps: 1) the guess of the manifold of the pure states (to be that of Schrödinger's waves), and 2) the guess of the statistical interpretation (stating that the transition probabilities are determined by the scalar products). In attempts of generalizing quantum mechanics the problem of the pure states is not the main difficulty, because some natural imitations of the step 1) are possible. The most obvious of them arises if one starts from a certain generalized "Schrödinger equation" (linear or not) which admits a certain conserved quantity $e(\varphi) \geqq 0$, characterizing the solutions $\varphi$ and interpreted as a "total wave charge". In that case, one can define the set $\Phi$ of all "normalized" solutions $\varphi$ with $e(\varphi)=1$ : one might assume that the solutions $\varphi \in \Phi$ represent the pure states of certain hypothetical quanta.

The choice of the statistical interpretation is much less obvious. For waves obeying non-linear equations the scalar products, in general, are not conserved by the time evolution and so, they are not appropriate to define the transition probabilities. The question thus arises, which functions of the non-linear waves should substitute the orthodox quantities $|(\psi, \varphi)|^{2}$ ? One of advantages of the convex scheme, is that this question does not need to be answered a priori. Indeed, in the approach outlined in $\S 2$ the transition probabilities are not fundamental but secondary: it is enough to have the statistical figure in order to reconstruct every detail of the statistical interpretation. The problem which becomes now essential concerns $S$ as a whole: how can one reconstruct the shape of the statistical figure starting from some minimal physical information? In particular: how can one find the structure of the mixed states if it is assumed that the pure states obey a non-linear wave equation? In the axiomatic approaches the structure of $S$ was usually determined by some universal regularity arguments always leading to the Hilbert space form of $S$. A possibility of a more general construction has been noticed in [16]; it yields $S$ provided that the following two elements are given:

1) A topological manifold $\Phi$ of elements $\varphi, \psi, \ldots$ which are candidates to represent the pure states of a hypothetical system. The correspondence between the elements $\varphi \in \Phi$ and the pure states needs not to be one-toone: each pure state, in general, may be represented by a whole subclass of elements of $\Phi^{7}$. The topology on $\Phi$ should be compatible with the

${ }^{7}$ It is also no harm if $\Phi$ covers a submanifold of quantum states wider than just the set of the pure states. This may happen in case of superselection rules, when $\Phi$ is taken to be the unit sphere in a Hilbert space, but some elements of $\Phi$, because of the structure of observables, are a posteriori identified with mixed states of the system. (For an interesting discussion of that point the author is indebted to Dr. S. Woronowicz.) 
observable properties of the pure states. In what follows $\Phi$ is called a manifold of pure states.

2) A class of real continuous functions on $\Phi$ representing the observational data and called the observables.

The second of the construction elements quoted here differs from the traditional algebra of observables and deserves a special description.

\section{Observables}

The concept of an observable in the present day theory has a complex status. It stands for both the measurement operation and its numerical output. The mathematical entities used to unify these two aspects are the self adjoint operators with their spectral decompositions. Below, this "conceptual union" will be split. The operator entities will be reserved to represent the transition processes rather than the observation acts [16]. The idea of observable will be associated with a purely numerical result of a measurement mathematically represented by a $c$-number function of the pure states.

Definition. Given a manifold of pure states $\Phi$ an observable or a statistical quantity is any function $f: \Phi \rightarrow \mathbb{R}$ whose values $f(\varphi)$ are interpretable as the statistical averages on various pure states $\varphi \in \Phi$ of a certain quantum mechanical measurement.

Here, the quantum mechanical measurement stands for any experimental mechanism assigning real numbers to the members of a quantum ensemble. The limiting assumption is that the mechanism should be sensitive only to the properties of the single ensemble individuals and not to their correlations within the ensemble. Since no other limitations are present, the above introduced concept of observable is wider than the orthodox one: it stands not only for an average indication of a "perfect" measuring device composed of ideal filters (as in majority of papers adopting von Neumann's approach) but can also describe average effects shown by "imperfect" or "mixed" devices [8] or even by quite arbitrary macroscopic bodies sensitive to the presence of quanta and endowed with numerical scales (thus coinciding with a more general concept of effects introduced by Ludwig [14]). In spite of that generality it is not at all so that every function on the set of pure states is an observable. To the contrary, the structure of the present day theory is precisely due to the fact that the observables form a relatively narrow subclass of all functions on $\Phi$. Indeed, the manifold $\Phi$ here is the unit sphere in a Hilbert space and the statistical averages are all given by the scalar products $(\psi, A \psi)(\psi \in \Phi)$ where $A$ are the self-adjoint operators. Thus, in the orthodox theory only the quadratic forms are observables: the other functions of $\psi$, though they can be experimentally determined, 
are not statistical averages of any quantum mechanical experiment. The above distinction is one of intriguing features of orthodox quantum mechanics: there is some mystery in the fact that for a microsystem certain functions of the pure states can and certain cannot be measured as statistical averages.

Since this is so, it is reasonable to assume that a similar distinction should also exist in case of a general theory. This fact is so essential that it might be choosen a new fundamental aspect of quantum theory competitive to "quantum logic" and "algebra of observables". Given the set of pure states of a certain hypothetical system, the nature of the system should be characterized by indicating which functions on the pure states are the observables. From now on the class of these functions will be denoted $F$. Given the pure states, the contents of $F$ may serve to classify the theories: the richer the class of observables than "more classical" the theory. Some general statements concerning the structure of $F$ can be made. Thus, $F$ should be a linear class: given two devices destinated to measure two observables $f_{1}, f_{2} \in F$, a new device can easily be constructed which would measure, as a statistical average, any linear combination $\lambda_{1} f_{1}+\lambda_{2} f_{2}\left(\lambda_{1}, \lambda_{2} \in \mathbb{R}\right)$. Because of the continuity of the macroscopic world some assumptions asserting the closed character of $F$ should also be adopted. It would be too strong to assume that any point-wise limit of functions from $F$ must also belong to $F$. This could lead to the appearence in $F$ of some discontinuous functions of the pure state corresponding to over-idealized measuring devices inconsistent with the "physical topology" (see also the discussion by Giles [8]). In what follows, a weaker property will be only assumed, namely, that $F$ is closed in the set $C(\Phi)$ of all real continuous function on $\Phi$ endowed with the topology of the point-wise convergence. This property will be called the relative closure of $F$.

The possibility of describing quantum systems in terms of observables suggests the following formal definition.

Definition. A quantum system without dynamics is a pair of entities $(\Phi, F)$ where $\Phi$ is a manifold of pure states and $F$ is a relatively closed, linear class of continuous real functions on $\Phi$ called "observables".

Before exhibiting the relation with the convex scheme of $\S 2$ it is worth while to notice that this definition is naturally suited to the particulae task of constructing non-linear variants of quantum mechanics. In fact, suppose that the manifold of pure states $\Phi$ coincides with a certain set of "non-linear waves". The most natural next heuristic step then is not so much to guess the character of quantum logic or the shape of $S$ but rather to postulate the class of observables $F$. Here, some natural hints exist based on the character of the non-linear wave dynamics. One feels, that a class of functions should be found which would reflect 
the structure of a non-linear wave equation as naturally as the class of the quadratic forms reflects that of linear equations.

The derivation of the observables from the dynamics of the $\psi$-wave is to be treated in $\S 4$. Now, it will be shown that the description of a quantum system in terms of pure states and observables is complete: given the pair of entities $(\Phi, F)$, the statistical figure of the system can be uniquely constructed.

\section{Construction of $S$}

Indeed, suppose that $\Phi$ and $F$ are known. Then, consider the set $\Pi$ of all the probability measures $\pi$ defined on Borel subsets of $\Phi$ for which the integrals $\int_{\Phi} f(\varphi) d \pi(\varphi),(f \in F)$, are convergent. The set $\Pi$ has an innate structure of a convex set: given two probability measures $\pi_{1}, \pi_{2} \in \Pi$ and two numbers $p_{1}, p_{2} \geqq 0, p_{1}+p_{2}=1$, the linear combination $p_{1} \pi_{1}$ $+p_{2} \pi_{2}$ is again a probability measure and it belongs to $\Pi$. The elements of $\Pi$ have a natural physical interpretation. Every $\pi \in \Pi$ is a prescription for preparing a mixed state: the prescription sais that for any Borel subset $\Omega \subset \Phi$ the fraction of the pure components from $\Omega$ taken into the mixture is $\pi(\Omega)$. Thus, $\Pi$ might be used to label the mixed states of the system. However, the correspondence between the elements $\pi \in \Pi$ (prescriptions) and the resulting mixtures is not necessarily one-to-one: this still depends upon the observable properties of the mixed states. For any $f \in F$ and $\pi \in \Pi$ the value of the observable $f$ on the mixed state prepared according to the prescription $\pi$ may be defined by the integral $f(\pi)=\int_{\Phi} f(\varphi) d \pi(\varphi)$. The numbers $f(\pi)(f \in F)$ represent the collection of the observable properties of the mixture $\pi$. Now, two measures $\pi, \pi^{\prime}$ are called equivalent $\left(\pi \equiv \pi^{\prime}\right)$ iff $f(\pi)=f\left(\pi^{\prime}\right)$ for every $f \in F$. Any two equivalent measures are interpreted as two prescriptions for producing mixtures which lead to physically indistinguishable mixed states. The equivalence relation $\equiv$ is a crucial element of the construction which accounts for the "first impossibility principle". Having given that relation one now defines a state (pure or mixed) as a class of equivalent probability measures. Consistently, one constructs the statistical figure $S$ as the convex set of all equivalence classes $S=\Pi / \equiv$.

While $\Pi$ is a generalized simplex, the quotient set $S$, in general, has a distinct structure: its geometry reflects the physics of the hypothetical quanta. Having given $S$ one can reconstruct the whole rest of the scheme as described above. This shows that the class of observables $F$ is indeed the key element of the theory which provides a unique construction of the generalized quantum scheme. A particular example of this construction accounts for the origin of the orthodox quantum mechanics. 


\section{Orthodox Theory}

That theory borrows some essential features from the linear classical field theory. Its main assumption states that each pure ensemble of quanta can be described by a complex field $\psi$ (a "wave function") which obeys a linear wave equation. Since for the linear equations the densities of the typical conservative quantities are quadratic in the field, it is natural to assume that one such quadratic density (which will be denoted by $\left.\psi^{+}(\boldsymbol{x}) \psi(\boldsymbol{x})\right)$ represents the average space density of the ensemble and the corresponding integral $(\psi, \psi)=\int_{\infty} \psi^{\dagger}(\boldsymbol{x}) \psi(\boldsymbol{x}) d_{3} \boldsymbol{x}$ is the total expected number of the ensemble particles. Since the total particle number does not enter into the description of a state, one can thus label the pure states by those $\psi$-fields for which $(\psi, \psi)=1$ : this leads to the manifold $\Phi$ being the unit sphere in a Hilbert space. At this moment certain elements of the statistical interpretation are already clear: it is decided that the probability of finding an average ensemble particle in an arbitrary space domain $\Omega$ is given by the quadratic form $p_{\Omega}(\psi)=\int_{\Omega} \psi^{\dagger}(\boldsymbol{x}) \psi(\boldsymbol{x}) d_{3} \boldsymbol{x}$. This assumption will be further called the primitive statistical interpretation and the forms $p_{\Omega}$ will be called the primitive observables. In agreement with the previous consideration the construction of the statistical figure $S$ requires the knowledge of the total class of observables $F$. The main indication as to the nature of that class follows again from the linearity of the quantum mechanical evolution equation. As seems reasonable to assume, the general quantum mechanical measurement upon $\psi$-wave can be accomplished in two stages. First, the $\psi$-wave undergoes a preliminary evolution process which is mathematically described by a certain norm conserving operation $\psi \rightarrow T \psi$. Because of the linearity of the evolution equation, the operation $T$ too is linear. Second, some primitive observables are measured upon the evolved wave $\psi^{\prime}=T \psi$. This is done by capturing the particle in one of disjoint reception domains $\Omega_{1}, \Omega_{2}, \ldots$ characterizing the structure of the measuring apparatus.

If the reception domains $\Omega_{j}$ are labelled by real numbers $\lambda_{j}$ (the scale of the apparatus) the statistical quantity measured is $f=\lambda_{1} p_{\Omega_{1}}^{\prime}$ $+\lambda_{2} p_{\Omega_{2}}^{\prime}+\cdots$, where $p_{\Omega}^{\prime}$ are the "evolved" primitive observables $p_{\Omega}^{\prime}(\psi)$ $=p_{\Omega}(T \psi)$. Now, since $p_{\Omega}$ are quadratic and $T$ is linear, the evolved $p_{\Omega}^{\prime}$ are again quadratic and so is the statistical quantity $f$. This implies that only the quadratic forms are measured as statistical quantities in quantum mechanical experiments. A question now arises as to how large is the set of quadratic forms which are observables? The simplest assumption is, that each real, continuous quadratic form on $\Phi$ is an observable and can be measured, at least in principle, as a statistical average of an adequate experiment. (A motivation of that assumption 
will be given in $\S 4$.) With these assumptions made the whole scheme of the orthodox theory can be uniquely deduced. Indeed, let $\pi$ be a probability measure on the unit sphere $\Phi$ and let $f$ be an observable. Since $f$ is quadratic, there exists a linear functional $f$ defined and continuous on a subset of the tensor product space $\overline{\mathscr{H}} \otimes \mathscr{H}$, such that $f(\psi)=\boldsymbol{f}(|\psi \times \psi|)$ $(\psi \in \Phi)$. Hence, $f(\pi)$ is determined by values of $\boldsymbol{f}$ on $\widehat{\mathscr{H}} \otimes \mathscr{H}$ :

$$
f(\pi)=\int_{\Phi} f(\psi) d \pi(\psi)=\int_{\Phi} \boldsymbol{f}(|\psi \times \psi|) d \pi(\psi)=\boldsymbol{f}\left(\int_{\Phi}|\psi \times \psi| d \pi(\psi)\right) .
$$

This formula implies that the physically essential properties of the mixture produced according to the prescription $\pi$ depend only upon the following hermitean, positive, unit-trace element of $\overline{\mathscr{H}} \otimes \mathscr{H}$ called a "density matrix":

$$
x_{\pi}=\int_{\Phi}|\psi \times \psi| d \pi(\psi) .
$$

This is how the orthodox "density matrices" emerge from the division of $\Pi$ into equivalence classes. The resulting statistical figure $S$ is isomorphic with the convex set of the entities of form (3.2): $S=\{x \in \mathscr{C}(\mathscr{H}): x$ $\left.=x^{\dagger} \geqq 0, \operatorname{Tr} x=1\right\}$. The rest of the orthodox scheme follows. The whole structure is essentially conditioned by the choice of $F$ : one might define the orthodox quantum mechanics as a theory of such a $c$-number wave for which only the quadratic forms are the observables. If another class of observables were choosen, different from that of the quadratic forms, the same mathematical mechanism would produce a distinct statistical figure corresponding to a different theory. In order to illustrate that dependence, a sequence of hypothetical schemes will be now discussed.

\section{Higher Order Schemes}

Similarly as before, let $\Phi$ be the unit sphere in $\mathscr{H}$. Now, however, assume that the class of observables $F$ is not the set of the quadratic forms like in orthodox theory but the set $F_{2 n}$ of all the continuous $2 n$-th order forms $f$ given by

$$
f(\psi)=h(\psi, \ldots, \psi ; \psi, \ldots, \psi),
$$

where $h\left(\varphi_{1}, \ldots, \varphi_{n} ; \psi_{1}, \ldots, \psi_{n}\right)$ are hermitean multiforms in $\mathscr{H}$ linear in the variables $\psi_{j}$ and antilinear in $\varphi_{j}^{\prime}$ 's. Since $(\psi, \psi)=1$ on $\Phi$, each $2 n$-order form $f \in F_{2 n}$ coincides on $\Phi$ with some forms of higher order: $f(\psi)$ $=(\psi, \psi)^{k} f(\psi)(k=1,2, \ldots)$. Hence, $F_{2} \subset F_{4} \subset F_{6} \ldots$ and so, the classes of observables $F_{2 n}$ correspond to hypothetical theories with extending varieties of macroscopic measuring devices. A characteristic property of such a sequence of theories is a step-wise recess of the "first impossibility": 
the wider the class of observables the more selective the perception of the mixed ensembles and more kinds of mixture become physically distinguishable.

Denote now by $S(\mathscr{H}, 2 n)$ the statistical figure constructed for $F=F_{2 n}$. An analytic description of $S(\mathscr{H}, 2 n)$ is possible similar to that employed by the orthodox theory. Indeed, suppose that $f$ is a $2 n$-order form. Then, there exists a linear, hermitean form $f$, defined and continuous on a subset of $\overline{\mathscr{H}} \underbrace{\otimes \cdots \otimes}_{n} \overline{\mathscr{H}} \otimes \mathscr{H} \underbrace{\otimes \cdots \otimes}_{n} \mathscr{H}$ such that $f(\psi)=f(\bar{\psi} \otimes \cdots \otimes \bar{\psi}$

$\otimes \psi \otimes \cdots \otimes \psi)(\psi \in \Phi)$. Hence, for any $\pi \in \Pi$ :

$$
\begin{aligned}
f(\pi) & =\int_{\Phi} f(\psi) d \pi(\psi)=\int_{\Phi} \boldsymbol{f}(\bar{\psi} \otimes \cdots \otimes \bar{\psi} \otimes \psi \otimes \cdots \otimes \psi) d \pi(\psi) \\
& =\boldsymbol{f}\left(\int_{\Phi} \bar{\psi} \otimes \cdots \otimes \bar{\psi} \otimes \psi \otimes \cdots \otimes \psi d \pi(\psi)\right) .
\end{aligned}
$$

This formula implies that the physical properties of the mixture prepared according to the prescription $\pi$ are fully determined by the following hermitean, positive, unit-trace element of $\overline{\mathscr{H}} \otimes \cdots \otimes \overline{\mathscr{H}}$ $\otimes \mathscr{H} \otimes \cdots \otimes \mathscr{H}$ which is a natural generalization of the orthodox "density matrix":

$$
\underset{2 n}{x_{n}}=\int_{\Phi} \bar{\psi} \otimes \cdots \otimes \bar{\psi} \otimes \psi \otimes \cdots \otimes \psi d \pi(\psi)
$$

The entity (3.5) might be called a "density tensor". The $S(\mathscr{H}, 2 n)$ is precisely the convex set of all density tensors of form (3.5) (the subset of all the positive, unit-trace elements of $\overline{\mathscr{H}} \otimes \cdots \otimes \mathscr{H} \otimes \cdots$ which are decomposable into convex integrals of the simple multitensors $\bar{\psi} \otimes \cdots \otimes \bar{\psi} \otimes \psi \otimes \cdots \otimes \psi)$. One thus arrives here at a new realization of the old scheme of quantum states, quantum observables and expectation values: the states are now the decomposable density tensors of an even order, the observables are arbitrary hermitean tensors of that same order and the expectation values are given by the tensor contractions.

The generalization introduced by $S(\mathscr{H}, 2 n)$ 's is formally similar to that achieved by the introduction of higher order multipole moments to the description of a density distribution. In fact, what the orthodox theory amounts to is the description of the probability measures $\pi \in \Pi$ in terms of their hermitean quadrupole moments with respect to the centre of the unit sphere $\Phi$. In case of $S(\mathscr{H}, 2 n)(n>1)$ higher order multipole moments of the measures are used. Since for the measures distributed over the unit sphere the higher order moments determine the lower order moments, the information contained in the subsequent "density tensors" is increasingly precise. Consistently, the statistical 
figures $S(\mathscr{H}, 2 n)$ are "increasingly classical": each next of them represents, from the point of view of each previous, a kind of a "hidden parameter" scheme with an increased manifold of the mixed states and recessed impossibility principles. This leads to a temptating question: is the impossibility of measuring as statistical average of anything but quadratic forms of $\psi$ indeed so fundamental as assumed by the present day quantum mechanics or is it only a technical barriere? Are the higher order forms of $\psi$ basically beyond the reach of quantum statistics or, perhaps they could be measured if only sufficiently subtle experimental techniques were employed?

\section{Forbidden Measurements}

Though no indications exist about the incompleteness of the present day theory, it is one of advantages of the "convex" approach that it exhibits some areas in which that theory, at least in principle, could be broken. Since the quadratic character of the observables is conditioned by the linearity of the evolution processes the most obvious such area consists in hypothetical evolution processes in which the quantum mechanical wave function would undergo a non-linear change. The most formal way of introducing such processes would be to assume some new couplings between the wave function and the external world. Thus, for instance, having a spinorial wave $\psi$ of Dirac electron one might assume the existence of a hypothetical external scalar field $\varphi$ coupled with $\psi$ according to $L_{\text {int }}=G(\bar{\psi} \psi) \varphi\left(G^{\prime} \neq\right.$ const $)$ and making $\psi$ to evolve according to the non-linear equation

$$
\left(\gamma^{\mu} \partial_{\mu}-\operatorname{im}\right) \psi+\varphi G^{\prime}(\bar{\psi} \psi) \psi=0 .
$$

The evolution (3.6), though allowed by the general principles of constructing couplings between $c$-number fields, would nevertheless break the consistency of the orthodox quantum mechanics. In fact, if an external field $\varphi$ was created catalyzing a non-linear behaviour of $\psi$, this field could be used to measure, as statistical averages, some nonquadratic forms of $\psi$ : in order to do that it would be sufficient to let a pure electron beam pass an external field $\varphi$ and then measure, upon the evolved $\psi$, some conventional quantum mechanical observables. As a result, the orthodox impossibility principles of quantum mechanics would be broken and the traditional manifold of the "density matrices" would become insufficient to represent the enriched set of the mixed states. Hence, if one assumes the sufficiency of the orthodox scheme, one must also assume the impossibility of non-linear response processes 
like (3.7). This exhibits a limitation of quantum theory close to Haag's consistency requirement: the orthodox electron cannot enter into arbitrary couplings without loosing its identity.

The above example is of rather theoretical merit: it seems not probable that quantum mechanics will be broken just by inventing new types of external fields. However, the non-linear processes might arise from less artificial sources. The partial differential equations of quantum mechanics (like those of Schrödinger or Dirac) concern, in essence, only such evolution processes in which a microparticle interacts with an infinitely heavy macroscopic surrounding which is a not affected by the presence of the particle. The processes of that kind form the proper domain of quantum mechanics and are well described by the known types external potentials none of which violates the traditional linearity. The situation is less obvious if the microparticle interacts with an object which, though macroscopic, is not infinitely inert but "subtle" and can modify its properties under the influence of the approaching particle. In that case one is tempted to consider the possibility of a hypothetical interaction process in which the wave function of the micro-object would undergo a non-linear change. The process may be described as follows.

There are two objects participating in the interaction: a microparticle and a macroscopic system. The microparticle is described by a certain wave function whereas the state of the macrosystem is determined by a set of classical parameters. The state of the whole of micro + macro system is simply the pair of states of the systems components. At the beginning of the process the microparticle and the macroscopic medium do not yet influence each other: the state of the microparticle is given by a certain wave $\psi$ which, at least approximately, evolves according to a linear wave equation whereas the macrosystem is in a certain standard initial state. Then the mutual interaction starts: the state of the macrosystem (which has the "subtle" ability of reacting to the particle presence) is modified under the influence of the approaching particle. This, in turn, modifies the way how the particle propagates. Thus, the wave function of the microparticle interacts with itself by modifying its own macroscopic environment. At a certain conventionally choosen final moment the interaction is again insignificant: the microparticle is now in a new state $\psi^{\prime}$ which depends upon the initial state $\psi: \psi^{\prime}=A(\psi)$. The result of the microparticle self interaction via the macroscopic system is the non-linearity of the operator $A$.

Note, that such theoretical schemes find some concrete realizations in the framework of the existing theory. Thus in the quasi-classical electrodynamics the electron is represented by a spinorial wave $\psi$ which is supposed to interact with a classical electromagnetic field. The wave $\psi$ here is assumed to produce a classical field $A_{\mu}$ which, in turn, influences 
the propagation of the wave:

$$
\begin{gathered}
A_{\mu}(x)=\int_{\infty} \Delta^{\mathrm{ret}}\left(x-x^{\prime}\right) j_{\mu}\left(x^{\prime}\right) d_{4} x^{\prime} ; \quad j_{\mu}=e \bar{\psi} \gamma_{\mu} \psi ; \\
{\left[\gamma^{\mu}\left(\partial_{\mu}-\frac{e}{c} A_{\mu}\right)-\operatorname{im} c\right] \psi=0 .}
\end{gathered}
$$

According to the orthodox quantum mechanics the schemes like the quasi-classical electrodynamics or the theory of a "self-consistent wave" cannot describe correctly the propagation of single quanta but can only provide approximate data concerning the average behaviour of clouds of many interacting particles. However, attempts are also made to assign to them an exact meaning $[13,20]$. According to that point of view, the electromagnetic field would be classical in nature and the quantal effects of electrodynamics would exclusively follow from the behaviour of fermions involved. Not entering into details of the discussion it is interesting to notice, however, that the serious assumption about the classical nature of the electromagnetic field would imply some deep changes in the quantum mechanics of fermions themselves. Indeed, the classical electromagnetic field interacting with the electron would be an example of a "subtle" macroscopic medium leading to a non-linear behaviour of the electron wave function $\psi$. This non-linearity, even if quantitatively small, could be arbitrarily amplified by adequate electron transmitters employing the classical character of $A_{\mu}$. One such hypothetical device is represented in Fig. 7.

The device on Fig. 7 is composed of an electromagnet and a sensor registering the intensity of the electric field at a detection point $P$. When the electric field at $P$ is above a certain critical value, the electromagnet responds connecting its own magnetic field; otherwize it remains inactive. Obviously, the device reacting in this manner could produce a strongly non-linear transformation of the electron $\psi$-wave and so, it would allow one to get out of the "enchanted circle" of the quadratic observables $^{8}$. An intriguing question thus returns: are the non-linear response processes of the quantum mechanical wave function indeed impossible? Or, perhaps, they could be produced if the experimental techniques were advanced enough to construct some "subtly reacting" macroscopic devices as that suggested in Fig. 7 ?

${ }^{8}$ By employing the classical character of $A^{\mu}$ one would be able to construct a large family of non-orthodox measuring devices based on the detection of the electromagnetic field. For instance: a counter which clicks only when the value of $\psi^{\dagger} \psi$ at a certain detection point is great enough; a counter which may not register the electron at a point $A$ if $\psi^{\dagger} \psi$ at another point $B$ is too big, etc. 

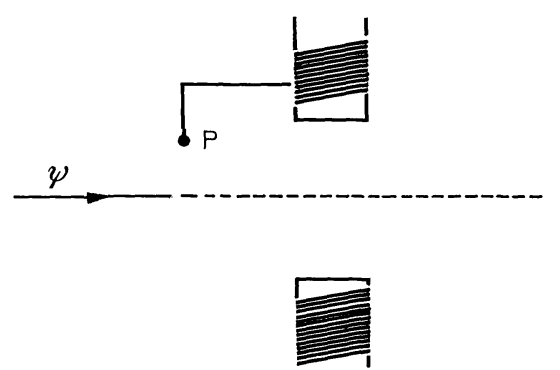

Fig. 7

From the point of view of the present day theory the answer is decisively negative. However, it must be replied that the orthodox theory should not be used to prove the unphysical character of non-linear response processes in quantum mechanics. It may not be excluded that the scheme of the modern theory of coupled systems with its formalism of tensor products (which is the alternative of non-linearity) plays a similar role in particle physics as the epicycle structure did in ancient kinematics. In that case the "forbidden processes" would not be impossible but to the contrary, they would form a natural area to look for new quantum phenomena.

\section{Dynamics and Geometry}

The scheme of the generalized quantum mechanics begins now to emerge. As is clear from the second impossibility principle, there is a link between the dynamics and the geometry of quantum systems (see discussion in $\S 2$ ). As follows from the considerations of $\S 3$ an important element of that link is the class of observables $F$. Now, the link will be completed by constructing the class of observables for systems with given dynamics.

Similarly as in $\S 3$ a hypothetical system will be considered with the manifold of pure states $\Phi$ being the set of all "normalized waves" $\psi=\{\psi(\boldsymbol{x})\}$, where the values $\psi(\boldsymbol{x})\left(\boldsymbol{x} \in \mathbb{R}^{3}\right)$ belong to a certain finite dimensional real or complex vector space $\Xi$ and the normalization condition is given by $e(\psi)=\int_{\infty} I(\psi(\boldsymbol{x})) d_{3} \boldsymbol{x}=1, I(\xi)$ being a positive functional of the vector variable $\xi \in \Xi$. For so normalized waves the preliminaries of the statistical interpretation will be defined by the quantities $p_{\Omega}(\psi)=\int_{\Omega} I(\psi(\boldsymbol{x})) d_{3} \boldsymbol{x}$ which will be called the primitive $o b$ - 
servables and interpreted as the probabilities of localizing the system in a pure state $\psi$ in various space domains $\Omega \subset \mathbb{R}^{3}$.

Given the primitive observables, the knowledge of dynamics happens to be the only element necessary to reconstruct the total class of observables $F$ and so, to determine the shape of the statistical figure.

\section{Dynamics}

In classical field theory the dynamics is usually introduced by postulating an evolution equation which governs the propagation of the fields. This point of view must be now modified. What, in fact, is the dynamics is not so much one evolution law but rather a whole family of such laws, determining the behaviour of the system in various "external environments". Indeed, what one deals with in case of Schrödinger or Dirac dynamics are whole families of structurally similar partial differential evolution equations with arbitrary potentials representing the "external world": it would be dynamically empty if one knew only the vacuum versions of the Schrödinger or Dirac equations. This leads to the following notion of dynamics as a complex entity.

Definition. An evolution law is any law (equation) which allows one to reconstruct the evolution of a physical system if the initial conditions are given. The dynamics is a class of evolution laws defining the behaviour of the system in various external conditions.

The concepts used here are open to further specifications. Thus in non-relativistic theories the initial conditions define the state of the system in a certain initial time moment and the knowledge of the evolution in the knowledge of the system states in all other moments. In the relativistic case the initial conditions stand for the Cauchy data defining the properties of the system on a certain space like surface and the knowledge of the evolution is the possibility of reconstructing the system behaviour on the whole rest of the space time. It is also open how rich a variety of data should be substituted for the external conditions. One might be interested in simplified models of dynamics with relatively poor classes of external conditions (as, for instance, the dynamics of a wave diffracting on a rigid, macroscopic body). In practice, more complex models of dynamics are important with the external reality described by at least arbitrary potentials. Some examples are listed in Table $1^{9}$.

9 For the non-linear equations of Table 1 the problem about the precise shape of the corresponding dynamics is still open. It is to be decided whether the theory should deal with the singular solutions, which are likely to appear in case of non-linear wave equations, or, perhaps, it may be restricted to the regular $\psi$-waves at the cost of limiting the external potentials and the initial conditions involved. 
Table 1

\begin{tabular}{llll}
\hline & $\begin{array}{l}\text { External } \\
\text { Potentials }\end{array}$ & Dynamics & Normalization \\
\hline 1 & $V$ & $i \hbar \frac{\partial \psi}{\partial t}=-\frac{\hbar^{2}}{2 m} \Delta \psi+V \psi$ & $\int_{\infty}|\psi|^{2} d_{3} x=1$ \\
2 & $V$ & $i \hbar \frac{\partial \psi}{\partial t}=-\frac{\hbar^{2}}{2 m} \Delta \psi+V|\psi|^{2} \psi$ & $\int_{\infty}|\psi|^{2} d_{3} x=1$ \\
3 & $V, U$ & $i \hbar \frac{\partial \psi}{\partial t}=-\frac{\hbar^{2}}{2 m} \Delta \psi+V \psi+U|\psi|^{2} \psi$ & $\int_{\infty}|\psi|^{2} d_{3} x=1$ \\
4 & $V$ & $i \hbar \frac{\partial \psi}{\partial t}=-\frac{\hbar^{2}}{2 m} \Delta\left(|\psi|^{2} \psi\right)+V \psi$ & $\int_{\infty}|\psi|^{4} d_{3} x=1$ \\
5 & $V$ & $i \hbar \frac{\partial \psi}{\partial t}=-\frac{\hbar^{2}}{2 m} \Delta\left(|\psi|^{2} \psi\right)+V|\psi|^{2} \psi$ & $\int_{\infty}|\psi|^{4} d_{3} x=1$ \\
\hline
\end{tabular}

All the examples of Table 1 are the generalizations of the nonrelativistic Schrödinger's dynamics which occupies the Position 1. The Example 2 has the vacuum propagation law identical with the Schrödinger vacuum equation but represents a distinct case of dynamics because the field $\psi$ is coupled distinctly to the external world. The dynamics of Example 3 is essentially richer than those of Example 1, 2 for it assumes an external reality in which two different types of potentials are present. In all three Examples 1-3 the quadratic form $\int_{\infty}|\psi|^{2} d_{3} x$ is the basic conservative quantity used to define the normalization and thus suggesting the coice of the primitive observables. It is no longer so in case of the Examples 4 and 5 which are based on the different vacuum propagation law:

$$
i \hbar \frac{\partial \psi}{\partial t}=-\frac{\hbar^{2}}{2 m} \Delta\left(|\psi|^{2} \psi\right)
$$

For that law the 4-th order form $\int_{\infty}|\psi|^{4} d_{3} x$ plays an analogous role as $\int_{\infty}|\psi|^{2} d_{3} x$ in case of the Schrödinger equation. Hence, if Examples 4, 5 reflected the dynamics of a certain quantum wave, the origins of the statistical interpretation could be based upon $|\psi|^{4}$ as a fundamental statistical density. Examples of wave dynamics for which the form $|\psi|^{k}(k>0)$ would be a basic conservative quantity could be as easily constructed. 


\section{Motion Group}

In what follows the decisive role belongs not to the dynamics itself but rather to a certain superstructure generated by the class of dynamical laws. In order to define it precisely the relative character of quantum states must be recalled. It is convenient to agree that the waves $\psi$ considered here represent the pure states in Schrödinger's picture. These are relative entities: by telling that a state of a system is given, one has in mind the situation which takes place on a certain space-like hyperplane $\Sigma$ in Minkowski (or Galileo) space-time as perceived by an inertial observer for whom $\Sigma$ is a plane of simultaneous events with the time coordinate $t=0$. Hence, the exact entity to which the concept of a (Schrödinger) state is refered is a space-like hyperplane endowed with a cartesianframe of three space-like coordinates and with the "past" and the "future" sides distinguished. Each such entity will be further called a framed hyperplane. In order not to complicate the scheme at this stage, all the framed hyperplanes considered below will be assumed to determine a common direction of the future in space-time.

Let now $\Sigma$ and $\Sigma^{\prime}$ be two framed hyperplanes and let the state of a hypothetical object on $\Sigma$ be described by a certain wave $\psi$. Assume, that the dynamics of the $\psi$-wave is known and that some definite external conditions in the space-time exist. Then, a definite dynamical law is valid which determines a new state on $\Sigma^{\prime}$ described by a certain wave $\psi^{\prime}$. Given the dynamics, the mapping $\psi \rightarrow \psi^{\prime}$ depends upon the pair of framed hyperplanes $\Sigma$ and $\Sigma^{\prime}$ and upon the external conditions involved: $\psi^{\prime}=T_{\Sigma, \Sigma^{\prime}, \text { Ext. }}(\psi)^{10}$.

The operators $T_{\Sigma, \Sigma^{\prime}, \text { Ext. }}: \Phi \rightarrow \Phi$ represent the possible evolutions of the $\psi$-waves (inbetween various pairs of framed hyperplanes and in presence of various external conditions) consistent with the dynamics assumed: they will be further called motions. The dynamical information essential for the structure of a quantum system can now be synthetized by introducing the following concept of a motion group which gatheres the totality of all could be motions of the system.

Definition. Given a manifold of pure state $\Phi$, the motion group $M$ is the smallest relatively closed group of transformations $\Phi \rightarrow \Phi$ con-

10 In case of dynamics like those of Table 1 the dependence $\psi \rightarrow \psi^{\prime}$ should be determined by solving the Cauchy problem for a partial differential equation. Given a wave $\psi=\{\psi(\boldsymbol{x})\}$ as an initial condition on the framed hyperplane $\Sigma$ and given the external potentials in the partial differential evolution equation one has to find put the unique space-time extrapolation $\{\psi(\boldsymbol{x}, t)\}$ of the initial wave $\psi$ such that $\psi(\boldsymbol{x}, 0)=\psi(\boldsymbol{x})$ (the coordinates $\boldsymbol{x}, t$ are induced by the frame on $\Sigma)$. The values $\psi(\boldsymbol{x}, t)$ at points $(\boldsymbol{x}, t) \in \Sigma^{\prime}$ after being transformed to the coordinate frame of $\Sigma^{\prime}$ (according to their covariant character) determine the required wave $\psi^{\prime}$. For the non-linear wave equations the mapping $\psi \rightarrow \psi^{\prime}$ may produce singular solutions on $\Sigma^{\prime}$ which might indicate that the theory should be opened toward singular Cauchy data. 


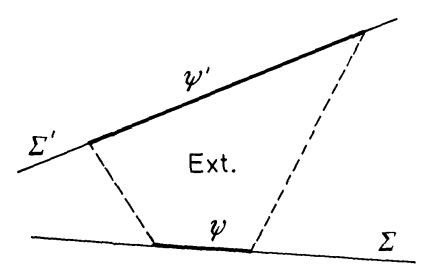

Fig. 8

taining all the operations $T_{\Sigma, \Sigma^{\prime}, \text { Ext. }}$. Here, the relative closure of $M$ means that every homeomorphism $\Phi \rightarrow \Phi$ which is a point-wise limit of transformations belonging to $M$ must also belong to $M$. Thus, the group $M$ is closed in the set of homeomorphisms of $\Phi$ endowed with its natural topology.

The above motion group is essentially wider than the sometimes employed dynamical group of a system. It contains all one-parameter transformation groups corresponding to all admissible turns of evolution. Since $\Sigma$ and $\Sigma^{\prime}$ can, in particular, be two distinctly framed versions of the same geometrical plane, the group $M$, appart of genuine dynamical processes, must also contain purely kinematical transformations of $\Phi$ corresponding to isometries on a fixed hyperplane in the Minkowski (or Galileo) space-time: it might be said that $M$ reflects the total "kineto-dynamical mobility" of the system.

The group $M$ provides an essential information concerning the structure of the observables. Indeed, having any measuring device destinated to measure a certain statistical average $f \in F$ one can produce more observables by alternating the measurement process. Instead of measuring straightforwardly the statistical average $f$ on a given wave $\psi \in \Phi$ one can let $\psi$ undergo first a certain preliminary kineto-dynamical process $T \in M$ and only afterwards measure $f$ on the evolved wave $\psi^{\prime}=T \psi$ thus obtaining a new statistical quantity: $(f T)(\psi)=f\left(\psi^{\prime}\right)$ $=f(T(\psi))$. In this way the existence of "motions" prevents one to assume too poor a class of observables: having any observable $f \in F$ one must also assume the existence in $F$ of infinity of other observables of form $f T$ generated by all possible evolution processes which the system might perform under the influence of various external forces. This suggests the following formal assumption:

Assumption. The class $F$ is invariant with respect to the motion group $M$.

With that assumption made, there is now only one heuristic step necessary to complete the generalized quantum scheme. 


\section{Main Assumption}

One of simplifications made by the present day theory consists in attributing an equal status to all the observables. Each self-adjoint operator in a Hilbert space is assumed to represent a physical observable: each of them is as well measurable as any other. It is a feature of quantum phenomenology, however, that is fails to reflect this abstract equality: for what one observes in reality is a distinguished role of the position measurements. Indeed, the known quantum experiments seem to follow the same general scheme in which the act of the localization is the ultimate experimentator's tool to extract the physical data ${ }^{11}$. The scheme consists in letting the particle wave function undergo a certain preliminary evolution process as a "preparatory stage" of the measurement and then in detecting the particle in one of spatially separated "reception domains".

As already noticed (compare $\S 3$ ) the statistical quantity measured in so arranged experiment is a linear combination of the "evolved" primitive observables $f=\sum_{j} \lambda_{j} p_{\Omega_{j}} T$ where $p_{\Omega_{j}}$ are the position observables corresponding to the space domains $\Omega_{j}\left(p_{\Omega}(\psi)\right.$ means the probability that a particle in a pure state $\psi$ will be localized in $\Omega$ and $T$ denotes the operator of the preliminary evolution process). This indicates that an arbitrary observable $f \in F$ can be either expressed or at least approached by linear combinations of the quantities $p T$ where $p$ are the primitive observables and $T$ are the motions of the system. The universality of this measurement technique does not seem to be conditioned by the particular character of the orthodox quantum mechanical evolution equations (like that of Schrödinger or Dirac) but it rather forms on immanent feature of the macroscopic measuring techniques. Hence, it is reasonable to believe that the reducibility of the general to the primitive observables should also exist in a general theory, though the operators $T$ are no longer linear there. This leads to the following general assumption which represents the required guess of the observables for a system with a non-linear dynamics.

Assumption. Given a manifold of pure states $\Phi$, a collection $P$ of real functions on $\Phi$ interpreted as the "primitive observables" and a motion group $M$, the complete class of observables $F$ is the smallest linear and relatively closed class of continuous real functions on $\Phi$ containing $P$ and invariant under the group $M$.

This assumption is the last heuristic step relating the geometry of quantum system to the dynamics assumed. Once this step is taken one has no longer freedom of specifying further the structure of quantum

11 The author appreciates a stimulating discussion of that point with Professor J. Werle. 
states and the statistical interpretation since those elements are already determined: having given $F$ one uniquely reconstructs the shape of the statistical figure and subsequently, all other geometric aspects of the theory. It is worth while to notice that from the heuristics just completed a certain abstract description of quantum systems emerges which is not even restricted to the particular domain of quantum theories based on wave equations.

\section{Group-Theoretical Model}

In fact, for the applicability of the constructions here outlined it is not essential to assume that the pure states correspond to certain $c$ number waves. Instead, one can take $\Phi$ to be a topological manifold of elements $\varphi, \psi, \ldots$ of arbitrary nature. The primitive statistical interpretation, too, does not need to depend upon the possibility of constructing positive conservative densities for some wave functions. Instead, one might just pick up a certain class $P$ of real functions on $\Phi$ and decrete them to be the "primitive observables" (the problem whether the elements $p \in P$ represent localization experiments and, eventually, how are they related to the space domains $\Omega \subset \mathbb{R}^{3}$ is to be resolved on the level of concrete theories). Finally, in order to fix the "dynamics" one has to choose a certain group $M$ of transformations $\Phi \rightarrow \Phi$ and decrete it to cover the totality of the kineto-dynamical processes which the system can undergo in various physical circumstances. This leads to the following group theoretical model of a quantum system.

Definition. A quantum system with dynamics is a set of five entities $(\Phi, M, P, F, S)$ where:

1) $\Phi$ is a topological manifold of points $\psi, \varphi, \ldots$ covering the pure states of a hypothetical physical system (the correspondence between the pure states and the elements $\varphi \in \Phi$, in general, is not one-to-one).

2) $M$ is a relatively closed subgroup of homeomorphisms $\Phi \rightarrow \Phi$ called the motion group and representing the dynamics of the system. (There is a generating subset of elements in $M$ which can be identified with the evolutions of the system in various external conditions.)

3) $P$ is a class of continuous functions on $\Phi$ which are called the primitive observables and interpreted as statistical averages of some "elementary measurements" which can be performed upon the system.

4) $F$ is the total class of observables constructed as the smallest linear and relatively closed class of real continuous functions on $\Phi$ which contains $P$ and is invariant under the transformation group $M$.

5) $S$ is the statistical figure constructed according to the prescription of $\S 3$. 
The intrinsic structure of quantum mechanics described by this definition might be represented in the following scheme.

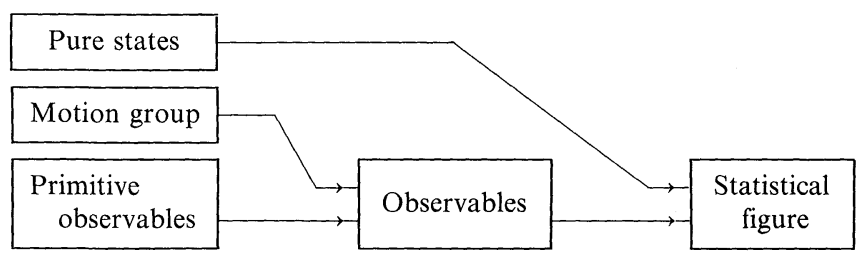

One of the most traditional realizations of that scheme is the nonrelativistic quantum mechanics of one particle.

\section{One-Particle Orthodox Quantum Mechanics}

For simplicity, a non-relativistic particle in one space dimension will be considered. The pure states then are represented by complex waves $\psi=\{\psi(x)\}\left(x \in \mathbb{R}^{1}\right)$ with the normalization $\int_{-\infty}^{+\infty}|\psi|^{2} d x=1$ and the manifold $\Phi$ is the unit sphere in the complex Hilbert space $\mathscr{H}$ $=L^{2}(-\infty,+\infty)$. The dynamical laws are of Schrödinger's form

$$
i \hbar \frac{\partial \psi}{\partial t}=-\frac{\hbar^{2}}{2 m} \frac{\partial^{2} \psi}{\partial x^{2}}+V(x) \psi
$$

where $V$ are arbitrary potentials. The quantity $|\psi|^{2}$ is a positive conservative density of the evolution laws (4.2); one thus guesses that it has the meaning of the probability density for the localization experiments (Born) and consistently, one chooses the primitive observables in the form:

$$
p_{\Omega}(\psi)=\int_{\Omega}|\psi|^{2} d x ; \quad \Omega \subset \mathbb{R}^{1} .
$$

The motion group of the system is now to be determined. This group should contain all the unitary one-parameter subgroups of form $U=e^{-\frac{i}{\hbar} H \tau}\left(\tau \in \mathbb{R}^{1}\right)$ where are the Hamiltonians with all possible external potentials: $H=-\frac{\hbar^{2}}{2 m} \frac{\partial^{2}}{\partial x^{2}}+V$. The further considerations form only an outline and have to be completed by exact proofs. Since the group $M$ 
is relatively closed, hence, together with any two families of operators $e^{\tau A}, e^{\tau B}$ (where $A$ and $B$ are imaginary operators in $\mathscr{H}$ ) it should also contain any operator of the form $e^{\alpha A+\beta B}$. This may be seen by applying the Trotter's formula:

$$
e^{\alpha A+\beta B}=\lim _{n \rightarrow \infty}\left(e^{\frac{\alpha A}{n}} e^{\frac{\beta B}{n}}\right)^{n} .
$$

Similarly, if $e^{\tau A}, e^{\tau B} \in M\left(\tau \in \mathbb{R}^{1}\right)$, the group $M$ should contain the unitary subgroup of the form $e^{\tau[A, B]}$ (provided that the commutator $[A, B]=A B-B A$ is well defined). This may be shown by employing the following approximation formula

$$
e^{[A, B]}=\lim _{n \rightarrow \infty}\left(e^{A / n} e^{B / n} e^{-A / n} e^{-B / n}\right)^{n^{2}} .
$$

These facts imply that the set of the imaginary operators $K$ for which $e^{\tau K} \in M$ for all $\tau \in \mathbb{R}^{1}$ forms a Lie algebra with respect to the commutation $[A, B]=A B-B A$. This algebra can be justly called the "Lie algebra" of the generalized "Lie group" $M$ and denoted $\operatorname{ad} M$. Now, taking $A=-\frac{\hbar^{2}}{2 m} \frac{\partial^{2}}{\partial x^{2}}$ and $B=-\frac{\hbar^{2}}{2 m} \frac{\partial^{2}}{\partial x^{2}}+V$, where $V$ is an arbitrary potential, one infers that $V=B-A \in \operatorname{ad} M$. Similarly, the operator $K_{1}=\frac{\partial}{\partial x} f(x)+f(x) \frac{\partial}{\partial x}=[A, B] \in \operatorname{ad} M$, where $f=-\frac{\hbar^{2}}{2 m} V^{\prime}$ is an arbitrary function. The $K_{1}$ happens to be the most general operator of form $K=k(x, p)$ which is linear in $p=-i \hbar \frac{\partial}{\partial x}$. In turn, by commuting $\frac{\partial^{2}}{\partial x^{2}}$ and $K_{1}$ one shows that $K_{2} \in \operatorname{ad} M$, where $K_{2}$ is the most general imaginary function $k(x, p)$ quadratic in $p$. By induction one shows that $\operatorname{ad} M$ contains any imaginary operator $k(x, p)$ which is analytic in $p$. Since $M$ is relatively closed, this indicates that ad $M$ coincides with the set of all imaginary operators in $\mathscr{H}$ and so, $M$ coincides with the unitary group acting on the unit sphere $\Phi$. The reconstruction of the class of observables $F$ now becomes the problem of the representation theory: the required $F$ is the smallest linear and relatively closed class of continuous real functions on $\Phi$ invariant under the unitary group and containing the quadratic forms $p_{\Omega}$. The known facts of the representation theory of the finite-dimensional unitary groups indicate that this class is just the set of all bounded quadratic forms on $\Phi$. The rest of the "genezis" of the orthodox theory was already described in $\S 3$.

Note, that the construction presented above exhibits some new consistency relations between the known elements of the orthodox quantum 
mechanics. Indeed, in the traditional presentation of that theory some essential elements of the statistical interpretation (such as the general expression for the transition probabilities by $\left.|(\psi, \varphi)|^{2}\right)$ are introduced in form of separate assumptions apparently independent of the primitive facts of the theory. This is not so in the group theoretical model just outlined. Here, once it is assumed that the wave $\psi$ obeys the Schrödinger's dynamics and once the Born's interpretation is accepted of $|\psi(x)|^{2}$ as the probability density, the whole rest of the scheme, together with the general expression for the transition probabilities, uniquely follows via construction of $S$. Thus, the group theoretical model yields a deepened insight into the "anatomy" of orthodox quantum mechanics.

\section{General Programme}

The possibility of more general realizations of the scheme is now open. An obvious thing to do would be to consider the known examples of classical non-linear wave dynamics with some naturally choosen "primitive observables" and then, to use the group theoretical model in order to see, to what kind of quantum systems do they lead. Mathematically, this involves a wide programme of structural investigations of non-linear field theories centered around two problems.

- One has to find out the motion groups for non-linear theories. This task, though it does not present a fundamental difficulty, is nontrivial as far as the effective knowledge of the motion group is concerned. As is already clear, for some non-linear versions of the Schrödinger's dynamics (like Examples 2, 3 in Table 1) the resulting motion group is significantly richer than the unitary group ${ }^{12}$. An essential question arises, as to, when $M$ coincides with the group of all homeomorphisms of the wave manifold $\Phi$ ? The theory in which this happens would lead to the class of observables $F$ coinciding with the set of all continuous real functions on $\Phi$ and so, the resulting statistical figure would be a generalized simplex. It would be interesting to know what kind of nonlinearities in the quantum mechanical wave equation imply, in this way, the return to a classical theory.

- Given a motion group $M$ acting on a wave manifold $\Phi$ and given a class of primitive observables $P$ one has to reconstruct the total class of observables $F$. Mathematically, this ammounts to looking for a class of continuous real functions on $\Phi$ which would be a minimal representation subspace of the group $M$ containing $P$. The effective solution of that problem requires the development of the representation theory of infinite dimensional Lie groups and the theory of special

\footnotetext{
12 Authors notes. Unpublished.
} 
functions on infinite dimensional differential manifolds. Those branches are already taking their first steps (see e.g. [7]); they form a natural area to look for the way out of the 50 years old formalism of Hilbert spaces in quantum physics.

\section{Consistency Problems}

Though the question about the practical use of the generalized quantum scheme is still open, this scheme already exhibits some essential consistency relations between quantum mechanics and other physical theories. This can be seen by applying consistency ideas of Haag's type to interacting physical systems.

Suppose, there are two generalized quantum systems $A$ and $B$. The phenomenology shows that the structure of $A$ can be described by a manifold of pure states $\Phi_{A}$ and a class of observables $F_{A}$. Similarly, $B$ can be described by a manifold of pure states $\Phi_{B}$ and a class of observables $F_{B}$. Suppose now, that a theory is formulated which tells, how $A$ interact with $B$. In this moment the problem of consistency arises. Indeed, when $A$ is assumed to interact with $B$ a certain new technique of measuring the statistical averages upon $A$-states is created. The techniques consists in letting the members of an ensemble of $A$-systems interact with replicas of $B$ and then in measuring some statistical quantities on the evolved $B$ instead of $A$.

Obviously, with the help of such a techniques any observable of $F_{B}$ induces a certain statistical average to be measured on the states of $A$. The question is: are these induced quantities already included in $F_{A}$ ? If so, the theory of interaction is consistent with the originally assumed structure of $A$. If not, the scheme is inconsistent and must be modified in at least one of three directions. Either one can interpret such a measurement as a sort of "subtle device" which yields a deeper insight into the real structure of $A$ and consistently, requires an extension of $F_{A}$. Or, one can infer that the assumptions concerning the structure of $B$ were premature and the consistency must be repaired by removing from $F_{B}$ some functions which are not indeed observables. Or, finally, one can suspect that there is something to be changed in the assumed interaction mechanism. A typical example of an inconsistent interaction scheme is that of quasi-classical electrodynamics described in $\S 3$. It seems, that there is no way how the classical electromagnetic field could be coupled with the orthodox electron without introducing too rich a class of observables, inconsistent with the orthodox structure of the electron states. This causes no difficulty in the present day electrodynamics where the mechanism of the inconsistency is removed by quantizing the electromagnetic field. The situation is much less obvious as far as the interaction 
between the microparticles and the gravitational field is concerned. Though some schemes with quantized gravitation are already emerging, the opinions also exist that the gravitation is an immanently classical phenomenon, even on the micro-level. If that was so, the consequences of that fact for quantum mechanics would be much deeper than generally recognized. Indeed, the classical gravitational field interacting with the electron would be a "subtly reacting medium" as described in $\S 3$ and the measuring devices based upon gravity detection would provide a method of measuring non-quadratic observables against one of fundamental prohibitions of the present day quantum mechanics. This might indicate, that we are facing the following alternative: either the gravitation is not classical or quantum mechanics is not orthodox. It is possible (though there is no mathematical proof at the moment) that, under the assumption about the classical nature of gravitation, the detectors of the gravitational field of a microparticle would allow one to measure, as a statistical average, any continuous functional of the microparticle pure state. This would lead to even stronger alternative: either the gravity is quantum or the electron is classical. These alternatives exhibit a somewhat peculiar situation of the present day theories: though it may be very difficult to quantize the gravitation, it is even more difficult not to do it. The incompleteness of the present day science at this point is, perhaps, one more reason why the scheme of quantum mechanics should not be prematurely closed.

Acknowledgements. The author is indebted to Professor I. Białynicki-Birula, Professor J. Hulanicki, Professor J. Plebański, Dr. R. Raczka, Professor A. Trautman, Professor J. Werle, Dr. L. Woronowicz and other collegues for their interest in this paper and helpful discussions. Special thanks are due to Professor F. A. E. Pirani for his hospitality at King's College in London in May and June 1971 where a part of this investigation has been carried. The author is also grateful to his colleagues from the Institute of Theoretical Physics of Chalmers in Göteborg and from the Institute of Theoretical Physics of the Royal Institute of Technology in Stockholm for critical and helpful discussions of nonlinearity problem in quantum mechanics.

\section{References}

1. Segal, I.E.: Ann. Math. 48, 930 (1947)

2. Birkhoff, G., von Neumann, J.: Ann. Math. 37, 823 (1936)

3. Dähn, G.: Commun. math. Phys. 9, 192 (1968); 28, 109 (1972); 28, 123 (1972)

4. Davies, E. B., Lewis, J. T.: Commun. math. Phys. 17, 239 (1970)

5. Davies, E. B.: Commun. math. Phys. 15, 277 (1969); 19, 83 (1970); 22, 51 (1971)

6. Edwards, C. M.: Commun. math. Phys. 16, 207 (1970); 20, 26 (1971); 24, 260 (1972)

7. Flato, M., Sternheimer,D.: Commun. math. Phys. 14, 5 (1969)

8. Giles, R.: J. Math. Phys. 11, 2139 (1970)

9. Gunson, J.: Commun. math. Phys. 6, 262 (1967)

10. Haag, R., Kastler, D.: J. Math. Phys. 5, 848 (1964) 
11. Hellwig, K.E., Kraus, K.: Commun. math. Phys. 11, 214 (1969); 16, 142 (1970)

12. Jauch, J.M., Piron, C.: Helv. Phys. Acta 42, 842 (1969)

13. Leiter, D.: Intern. J. Theor. Phys. 4, 83 (1971)

14. Ludwig, G.: Z. Naturforsch. 22a, 1303 (1967); 22a, 1324 (1967)

15. Ludwig, G.: Commun. math. Phys. 4, 331 (1967); 9, 1 (1968); 26, 78 (1972)

16. Mielnik, B.: Commun. math. Phys. 15, 1 (1969)

17. Neumann, H.: Commun. math. Phys. 23, 100 (1971)

18. Piron, C.: Helv. Phys. Acta 37, 439 (1964)

19. Pool, J.C.T.: Commun. math. Phys. 9, 118 (1968); 9, 212 (1968)

20. Sachs, M.: Intern. J. Theor. Phys. 4, 83 (1971)

21. Stolz,P.: Commun. math. Phys. 11, 303 (1969); 23, 117 (1971)

22. Gudder, S.: Commun. math. Phys. 29, 249 (1973)

Communicated by R. Haag

B. Mielnik

University of Warsaw

Institute for Theoretical Physics

Division of Mathematical Physics

Hòza 69

Warszawa, Poland

\section{Erratum}

Del Grosso, G.: On the Local Central Limit Theorem for Gibbs Processes. Commun. math. Phys. 37, 141-160 (1974)

The year in the copyright note on page 141 should read 1974 instead of 1973. 